\title{
BEST APPROXIMATION OF A NORMAL OPERATOR IN THE SCHATTEN $p$-NORM
}

\author{
RICHARD BOULDIN
}

\begin{abstract}
Let $A$ be a fixed normal operator and let $\varkappa(\Lambda)$ denote the normal operators with spectrum contained in $\Lambda$. Provided there is some $N$ in $\varkappa(\Lambda)$ such that $A-N$ belongs to the Schatten class $c_{p}, p>2$, the main result of this paper obtains a best approximation for $A$ from $\varkappa(\Lambda)$ with respect to the Schatten $p$-norm. A necessary and sufficient condition is given for $A$ to have a unique best approximation in that case.
\end{abstract}

1. Introduction. If $\Lambda$ is a closed nonempty set in the complex plane then $\Re(\Lambda)$ denotes the normal (bounded linear) operators on the fixed separable Hilbert space $H$ with spectrum contained in $\Lambda$. For any compact operator $T$ let $|T|=\left(T^{*} T\right)^{1 / 2}$ and let $s_{1}(T), s_{2}(T), \ldots$ be the eigenvalues of $|T|$ in nonincreasing order repeated according to multiplicity. If, for some $p>1$, one has

$$
\sum_{j=1}^{\infty} s_{j}(T)^{p}<\infty
$$

then one says that $T$ belongs to the Schatten class $c_{p}$ which is normed with

$$
\|T\|_{p}=\left(\sum_{j=1}^{\infty} s_{j}(T)^{p}\right)^{1 / p} .
$$

A good reference for the general theory of Schatten classes is [8]. The problem considered in this paper is to find a best approximation for a fixed normal operator $A$ from $\mathscr{T}(\Lambda)$ using the norm $\|\cdot\|_{p}$. The problem of determining when $A$ has a unique best approximation is also considered.

2. Main results. In [12] P. R. Halmos constructed a best approximation of the fixed normal operator $A$ from $\mathscr{T}(\Lambda)$ using the usual operator norm. In order to state his result it is necessary to discuss the class of complex valued functions of a complex variable which are called retracts. One says that $F(z)$ is a distance minimizing retract onto $\Lambda$ provided each $F(z)$ belongs to $\Lambda$ and

$$
|z-F(z)|<|z-\lambda| \text { for all } \lambda \text { in } \Lambda \text {. }
$$

Provided $\Lambda$ is closed and nonempty there is a Borel measurable distance minimizing retract onto $\Lambda$; see [12] for a nice proof. If $\Lambda$ is convex and nonempty then there is a unique distance minimizing retract; see [13, Theorem 7.8, p. 94]. For $A$ and $\Lambda$ as above, the theorem of Halmos in [12] asserts that

$$
\|A-F(A)\|<\|A-N\| \text { for every } N \in \Re(\Lambda),
$$

Received by the editors May 9, 1979 and, in revised form, August 24, 1979 and October 9, 1979. AMS (MOS) subject classifications (1970). Primary 47B20. 
where $F(z)$ is a Borel measurable distance minimizing retract onto $\Lambda$. Note $F(A)$ belongs to $\Re(\Lambda)$.

The main results are now stated; the proofs are given in the next section.

THEOREM 1. Let $A$ be a fixed normal operator with spectrum $\sigma(A)$. In order for there to exist some $N \in \Re(\Lambda)$ such that $A-N$ belongs to $c_{p}, p>2$, it is necessary and sufficient that $\sigma(A) \backslash \Lambda$ is a (possibly empty or possibly infinite) countable set of finite dimensional isolated eigenvalues $\left\{\alpha_{1}, \alpha_{2}, \ldots, \alpha_{l}\right\}$, repeated according to multiplicity, such that $\Sigma_{j}\left(\operatorname{dist}\left(\alpha_{j}, \Lambda\right)\right)^{p}$ is finite.

THEOREM 2. Let $A$ be a fixed normal operator and let $F(z)$ be a Borel measurable distance minimizing retract of the complex plane onto $\Lambda$. If there exists some $N \in \mathscr{T}(\Lambda)$ such that $A-N$ belongs to $c_{p}, p>2$, then $A-F(A)$ belongs to $c_{p}$ and

$$
\|A-F(A)\|_{p}<\|A-N\|_{p} .
$$

Furthermore, $F(A)$ is the unique choice of $N$ producing equality in (*) if and only if every point of $\sigma(A)$ has a unique closest point in $\Lambda$. In particular, if $\Lambda$ is convex then equality in (*) implies $N=F(A)$.

In the case that $A$ is an invertible nonnegative operator and $\Lambda$ is the unit circle, then the theorem was proved in [2] by means of Fréchet derivatives. It should be noted that if $F(z)$ is a distance minimizing retract onto the unit circle and $A$ is an invertible nonnegative operator then $F(A)$ is the identity operator. The reformulation of the result given in [2] shows that it extends theorems in [1], [6], [7] which are relevant to quantum chemistry. Also, [10, Lemma 3.1, p. 323] is a special case of the theorem.

The assertion in the theorem that $A-F(A)$ belongs to $c_{p}$ provides a remarkable contrast to previously known results about closure properties of $c_{2}$. Since $F(z)=z$ for every $z$ in $\Lambda, F(N)$ equals $N$ and the statement that $A-F(A)$ belongs to $c_{2}$ is equivalent to the statement that $F(A)-F(N)$ belongs to $c_{2}$. In [4] the best result of this type asserts that $f(V)-f(U)$ belongs to $c_{2}$ when $V-U$ belongs to $c_{2}, V$ and $U$ are unitary and $f(z)$ is a function on the unit circle with its derivative satisfying a Lipshitz condition.

Let $\Lambda=\{0,1\}$ and $A=(1 / 2) P$ where $P$ is the orthogonal projection onto some finite dimensional subspace of $H$. Then any orthogonal projection $R$ onto a subspace of the range of $P$ has the property that

$$
\|A-F(A)\|_{p}>\|A-R\|_{p}
$$

for $p>1$ and any retract $F(z)$ onto $\Lambda$. Thus, the uniqueness statement of the theorem is false without some additional hypothesis.

3. Proof of the main results. For the reader's convenience a proof to the following well-known lemma is included.

LEMMA 1. Let $A$ be a fixed normal operator. If there exists some $N \in \Re(\Lambda)$ such that $(A-N) \in c_{p}$ then the only points in the spectrum of $A$, denoted $\sigma(A)$, not contained in $\Lambda$ are isolated eigenvalues with finite multiplicity. 
Proof. Note that $A$ is a compact perturbation of $N$. According to Weyl's theorem for normal operators, $A$ and $N$ have the same Weyl spectrum. The reader can find a contemporary discussion of Weyl's theorem in [3]. For any normal operator $T$ the Weyl spectrum coincides with the points of $\sigma(T)$ which are not isolated eigenvalues with finite multiplicity. (See [5, Theorem 3] or [3, Theorem 5.1].) The operators for which the above set coincides with the Weyl spectrum are characterized in [11]. Since the Weyl spectrum of $N$-and, hence, the Weyl spectrum of $A$-is contained in $\Lambda$, the conclusion of the lemma follows.

LEMMA 2. If $N$ is a normal operator, $\alpha$ is some scalar and $e$ is some unit vector then

$$
\|(\alpha-N) e\|>\operatorname{dist}(\alpha, \sigma(N)) \text {. }
$$

If there is a unique point $\beta$ in $\sigma(N)$ which is closest to $\alpha$ and equality holds in (*) then $e$ is an eigenvector for $N$ and $\beta$ is the corresponding eigenvalue.

Proof. The proof of (*) given in [12] is incorporated in the following. Let $E(\cdot)$ be the spectral measure of $N$ and note that

$$
\begin{aligned}
\|(\alpha-N) e\|^{2} & =\int_{\sigma(N)}|\alpha-z|^{2} d\langle E(z) e, e\rangle \\
& >\int_{\sigma(N)} \operatorname{dist}(\alpha, \sigma(N))^{2} d\langle E(z) e, e\rangle \\
& =\operatorname{dist}(\alpha, \sigma(N))^{2} .
\end{aligned}
$$

Thus, (*) above holds.

Assume that equality holds in (*) and $\beta$ is the unique point of $\sigma(N)$ closest to $\alpha$. It follows that

$$
|\alpha-z|=\operatorname{dist}(\alpha, \sigma(N))
$$

or

$$
z=\beta
$$

almost everywhere with respect to the measure $\langle E(\cdot) e, e\rangle$. Thus, one has

$$
\|(N-\beta) e\|^{2}=\int_{\sigma(N)}|z-\beta|^{2} d\langle E(z) e, e\rangle=0
$$

and the lemma is proved.

Lemma 3. Let $T$ be in $c_{p}$ and let $\left\{e_{1}, \ldots, e_{l}\right\}$ be a (possibly infinite) orthonormal set. Then one has the inequality

$$
\left.\|T\|_{p}^{p}\right\rangle \sum_{j=1}^{l}\left\langle|T| e_{j}, e_{j}\right\rangle^{p}
$$

for $p>1$.

Proof. See [10, Item 5, p. 94].

LEMMA 4. Let $T$ be in $c_{p}, p>2$. If $\left\{e_{1}, e_{2}, \ldots\right\}$ is an orthonormal sequence then $\|T\|_{p}^{p}>\Sigma_{j}\left\|T e_{j}\right\|^{p}$. 
Proof.

$$
\begin{aligned}
\|T\|_{p}^{p} & =\||T|\|_{p}^{p}=\sum_{j} s_{j}(|T|)^{p} \\
& =\sum_{j} s_{j}\left(|T|^{2}\right)^{p / 2}=\left\||T|^{2}\right\|_{p / 2}^{p / 2} \\
& >\sum_{j}\left\langle|T|^{2} e_{j}, e_{j}\right\rangle^{p / 2} \text { by Lemma } 3 \\
& =\sum_{j}\left\|T e_{j}\right\|^{p} .
\end{aligned}
$$

It is worth noting that if $\left\{e_{j}\right\}$ is an orthonormal basis then $\|T\|_{2}^{2}=\Sigma_{j}\left\|T e_{j}\right\|^{2}$, while if $p=1$, the reverse inequality holds and may be strict: $\|T\|_{1}<\Sigma_{j}\left\|T e_{j}\right\|$.

Proof of Theorem 1. Note that Lemma 1 applies to $A$ and let $\left\{e_{1}, \ldots, e_{l}\right\}$ be a maximal orthonormal set of eigenvectors for $A$ corresponding to the isolated eigenvalues $\left\{\alpha_{1}, \ldots, \alpha_{l}\right\}$ of $A$ not contained in $\Lambda$. In order to show the inequality (*) one observes the following

$$
\begin{aligned}
\|A-N\|_{p}^{p} & >\sum_{j}\left\|(A-N) e_{j}\right\|^{p} \quad \text { by Lemma } 4 \\
& >\sum_{j} \operatorname{dist}\left(\alpha_{j}, \sigma(N)\right)^{p} \quad \text { by Lemma } 2 \\
& >\sum_{j} \operatorname{dist}\left(\alpha_{j}, \Lambda\right)^{p} .
\end{aligned}
$$

In order to prove the converse, write $A$ as $A_{1} \oplus A_{2}$ relative to the decomposition $H=E(\Lambda) H \oplus E\left(\Lambda^{c}\right) H$, where $E(\cdot)$ is the spectral measure of $A$ and $\Lambda^{c}$ means the complement of $\Lambda$. Note that $A_{1} \in \mathscr{T}(\Lambda)$ and $A_{2}=\Sigma_{j-1}^{l}\left\langle\cdot, e_{j}>\alpha_{j} e_{j}\right.$ where $\left\{e_{1}, \ldots, e_{l}\right\}$ is a maximal orthonormal set of eigenvectors for $A$ corresponding to $\left\{\alpha_{1}, \ldots, \alpha_{l}\right\}$. Note that

$$
F(A)=A_{1} \oplus F\left(A_{2}\right)=A_{1} \oplus \sum_{j=1}^{l}\left\langle\cdot, e_{j}\right\rangle F\left(\alpha_{j}\right) e_{j} \in \Re(\Lambda) .
$$

Also observe that

$$
\begin{aligned}
\|A-F(A)\|_{p}^{p} & =\left\|0 \oplus \sum_{j=1}^{l}\left\langle\cdot, e_{j}\right\rangle\left(\alpha_{j}-F\left(\alpha_{j}\right)\right) e_{j}\right\|_{p}^{p} \\
& =\sum_{j=1}^{l}\left|\alpha_{j}-F\left(\alpha_{j}\right)\right|^{p}=\sum_{j=1}^{l} \operatorname{dist}\left(\alpha_{j}, \Lambda\right)^{p}<\infty .
\end{aligned}
$$

Proof of Theorem 2. By Lemma 4 and Lemma 2, with the notation of the preceding proof, one obtains

$$
\begin{aligned}
\|A-N\|_{p}^{p} & \geqslant \sum_{j=1}^{l}\left\|(A-N) e_{j}\right\|^{p} \\
& \geqslant \sum_{j=1}^{l}\left\|\left(\alpha_{j}-N\right) e_{j}\right\|^{p}>\sum_{j=1}^{l} \operatorname{dist}\left(\alpha_{j}, \sigma(N)\right)^{p} \\
& =\sum_{j=1}^{l} \operatorname{dist}\left(\alpha_{j}, \Lambda\right)^{p}=\sum_{j=1}^{l}\left|\alpha_{j}-F\left(\alpha_{j}\right)\right|^{p}
\end{aligned}
$$


It will now be shown that the last sum is $\|A-F(A)\|_{p}^{p}$. Write $A$ as $A_{1} \oplus A_{2}$ relative to the decomposition $H=E(\Lambda) H \oplus E\left(\Lambda^{c}\right) H$, where $E(\cdot)$ is the spectral measure of $A$. Since $F(z)=z$ for all $z$ in $\Lambda$ one has

$$
F(A)=F\left(A_{1}\right) \oplus F\left(A_{2}\right)=A_{1} \oplus F\left(A_{2}\right) .
$$

Thus, if $\left\{f_{1}, f_{2}, \ldots\right\}$ is any orthogonal basis for $E(\Lambda) H$ then $\left\{e_{1}, \ldots, e_{l}, f_{1}, f_{2}, \ldots\right\}$ diagonalizes $A-F(A)$ and the corresponding eigenvalues are $\left\{\alpha_{1}-F\left(\alpha_{1}\right), \ldots, \alpha_{l}-F\left(\alpha_{l}\right), 0,0, \ldots\right\}$, respectively. It is now elementary that

$$
\|A-F(A)\|_{p}^{p}=\sum_{j=1}^{l}\left|\alpha_{j}-F\left(\alpha_{j}\right)\right|^{p}
$$

and, hence,

$$
\|A-N\|_{p}^{p}>\|A-F(A)\|_{p}^{p} .
$$

Assume that each point of $\sigma(A)$ has a unique closest point in $\Lambda$ and let $N$ be some operator from $\Re(\Lambda)$ for which equality holds in (*). Thus, equality holds throughout the inequalities of the first paragraph of this proof. In particular, using Lemma 2 , for $j=1, \ldots, l$, one has

$$
\left\|\left(\alpha_{j}-N\right) e_{j}\right\|=\operatorname{dist}\left(\alpha_{j}, \Lambda\right)=\operatorname{dist}\left(\alpha_{j}, \sigma(N)\right) .
$$

Lemma 2 shows that $e_{j}$ is an eigenvector for $N$ with corresponding eigenvalue $F\left(\alpha_{j}\right)$. Choosing $\left\{f_{1}, f_{2}, \ldots\right\}$ as in the second paragraph of this proof, one notes that Lemma 4 implies

$$
\|A-N\|_{p}^{p} \geqslant \sum_{j=1}^{l}\left|(A-N) e_{j}\right|^{p}+\sum_{j}\left\|(A-N) f_{j}\right\|^{p} .
$$

Since equality holds throughout the inequalities of the first paragraph of this proof, it must be that

$$
\left\|(A-N) f_{j}\right\|=0, \quad j=1,2, \ldots
$$

Thus, the restriction of $A$ and $N$ to $E(\Lambda) H$ coincide. Consequently the restrictions of $A, N$ and $F(A)$ coincide. Since $N$ and $F(A)$ coincide on closed span $\left\{e_{1}, \ldots, e_{l}\right\}$ $=E\left(\Lambda^{c}\right) H$, it is proved that $N=F(A)$.

In the event that $\Lambda$ is convex every point in the complex plane has a unique nearest point in $\Lambda$ and so the preceding proof shows that $N=F(A)$.

If there exists some $\lambda \in \sigma(A)$ such that $|\lambda-\mu|=|\lambda-F(\lambda)|$ and $F(\lambda) \neq \mu \in \Lambda$ then the definition of $F$ can be altered by setting $F(\lambda)=\mu$. Thus, there are two Borel measurable distance minimizing retracts onto $\Lambda$ which are different on $\sigma(A)$. This proves $F(A)$ is not the unique best approximation.

\section{REFERENCES}

1. J. G. Aiken, H. B. Jonassen and H. S. Aldrich, Löwdin orthonormalization as a minimum energy perturbation, J. Chem. Phys. 62 (1975), 2745-2746.

2. J. G. Aiken, J. A. Erdös and J. A. Goldstein, Unitary approximation of positive operators (preprint).

3. S. K. Berberian, The Weyl spectrum of an operator, Indiana Univ. Math. J. 20 (1970), 529-544.

4. M. S. Birman and M. Z. Solomyak, Stieltjes double-integral operators, Spectral Theory and Wave Processes (Topics in Mathematical Physics, vol. 1), Consultants Bureau, New York, 1976.

5. R. Bouldin, Essential spectrum for a Hilbert space operator, Trans. Amer. Math. Soc. 163 (1972), 437-445. 
6. B. C. Carlson and J. M. Keller, Orthogonalization procedures and localization of Wannier functions, Phys. Rev. 105 (1957), 102-103.

7. A. J. Coleman, Structure of fermion density matrices, Rev. Modern Phys. 35 (1963), 668-689.

8. N. Dunford and J. T. Schwartz, Linear operators, vol. II, Interscience, New York, 1963.

9. K. Fan, Maximum properties and inequalities for eigenoalues of completely continuous operators, Proc. Nat. Acad. Sci. U.S.A. 37 (1951), 760-766.

10. I. C. Gohberg and M. G. Krein, Introduction to the theory of linear nonselfadjoint operators, Transl. Math. Monographs, vol. 18, Amer. Math. Soc., Providence, R.I., 1969.

11. K. Gustafson, Necessary and sufficient conditions for Weyl's theorem, Michigan Math. J. 19 (1972), 71-81.

12. P. R. Halmos, Spectral approximants of normal operator, Proc. Edinburgh Math. Soc. 19 (1974), 51-58.

13. F. A. Valentine, Convex sets, McGraw-Hill, New York, 1964.

14. H. Weyl, Inequalities between the two kinds of eigenvalues of a linear transformation, Proc. Nat. Acad. Sci. U.S.A. 35 (1949), 408-411.

Department of Mathematics, University of Grorgi, Athens, Grorgu 30602 